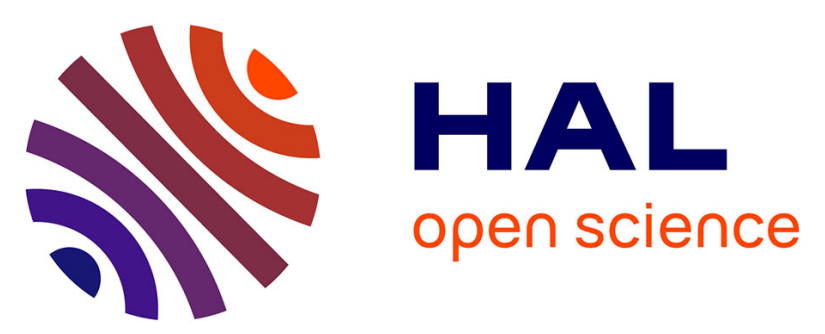

\title{
Implication of Water Molecules at the Silica - Ibuprofen Interface in Silica-Based Drug Delivery Systems Obtained through Incipient Wetness Impregnation
} Thierry Azaïs, Guillaume Laurent, Kuldeep Panesar, Andrei Nossov, Flavien Guenneau, Cristina Sanfeliu Cano, Corine Tourné-Péteilh, Jean-Marie Devoisselle, Florence Babonneau

\section{To cite this version:}

Thierry Azaïs, Guillaume Laurent, Kuldeep Panesar, Andrei Nossov, Flavien Guenneau, et al.. Implication of Water Molecules at the Silica - Ibuprofen Interface in Silica-Based Drug Delivery Systems Obtained through Incipient Wetness Impregnation. Journal of Physical Chemistry C, 2017, 121 (48), pp.26833-26839. 10.1021/acs.jpcc.7b08919 . hal-01637716

\section{HAL Id: hal-01637716 https://hal.sorbonne-universite.fr/hal-01637716}

Submitted on 17 Nov 2017

HAL is a multi-disciplinary open access archive for the deposit and dissemination of scientific research documents, whether they are published or not. The documents may come from teaching and research institutions in France or abroad, or from public or private research centers.
L'archive ouverte pluridisciplinaire HAL, est destinée au dépôt et à la diffusion de documents scientifiques de niveau recherche, publiés ou non, émanant des établissements d'enseignement et de recherche français ou étrangers, des laboratoires publics ou privés. 


\title{
Implication of Water Molecules at the Silica -
}

\section{Ibuprofen Interface in Silica-Based Drug Delivery}

\section{Systems Obtained through Incipient Wetness}

\author{
Impregnation
}

Thierry Azaïs, ${ }^{a} *$ Guillaume Laurent, ${ }^{a}$ Kuldeep Panesar, ${ }^{a}$ Andrë Nossov, ${ }^{a}$ Flavien Guenneau, ${ }^{a}$ Cristina Sanfeliu Cano, ${ }^{a} \dagger$ Corine Tourné-Péteilh, ${ }^{b}$ Jean-Marie Devoisselle ${ }^{b}$ and Florence Babonneau $^{a}$

${ }^{a}$ Sorbonne Universités, UPMC Univ Paris 06, CNRS, Collège de France, Laboratoire de Chimie de la Matière Condensée de Paris (LCMCP), Paris, France

${ }^{\text {b UMR }} 5253$ CNRS ENSCM UM, MACS, Institut Charles Gerhardt Montpellier, 1701 Place Eugene Bataillon, F-34095 Montpellier, France

Key words: Ibuprofen; Encapsulation; Drug delivery systems; solid state NMR 


\begin{abstract}
The dynamical behavior of ibuprofen or benzoic acid (model molecule of ibuprofen) encapsulated in mesoporous silica is very specific as a very high mobility is evidenced at ambient temperature due to the existence of confinement effect. In this contribution, we demonstrate through variable temperature ${ }^{1} \mathrm{H}$ MAS NMR experiments that this specific dynamical behavior is also related to a fast chemical exchange that takes place between protons of the $\mathrm{COOH}$ group of the organic molecule and protons from water molecules at the surface of the silica for materials obtained through incipient wetness impregnation. This phenomenon implies a weak interaction between the guest molecule and the silica surface that is related to the fast release profile of encapsulated ibuprofen observed in vitro.
\end{abstract}




\section{Introduction}

Confined molecules in porous matrices adopt a specific dynamical behavior because of confinement effect that implies a greater mobility of the trapped species when compared to molecules in bulk. This typical behavior is a consequence of a depression of the thermodynamical parameters including the phase transition temperatures. ${ }^{1,2}$ Thus, the encapsulated molecules can adopt a liquid-like behavior at room temperature, even if the pure substance is a solid under such conditions. This confinement effect, which is still not well understood, was often studied on simple liquids such as water, ${ }^{3,4}$ methanol, ${ }^{5,6}$ or benzene $^{7,8,9,10,11}$ in order to study their dynamical behavior and to determine the phase transition temperatures of the encapsulated molecules. The chosen porous matrices used for the encapsulation are often mesoporous silica such as SBA-15,9,10 or MCM-41..$^{5,6,10}$

Recently, the characterization of confined drug molecules became essential in the field of pharmaceutical studies due to a growing interest in mesoporous silica-based systems for the controlled release of hydrophobic drugs. ${ }^{12,13,14,15,16,17,18,19}$ The release kinetics depends on different parameters such as $(i)$ the chemical composition of the drug ${ }^{20}$ (ii) the $\mathrm{pH}$ of the dissolution medium for ionizable drugs, ${ }^{14}$ (iii) the pore diameter of the mesoporous matrix, ${ }^{16}$ (iv) the chemical composition of the silica surface, ${ }^{21}(v)$ the pore connectivity and its geometry, and (vi) the stability of the matrix ${ }^{15}$ in aqueous medium. Ibuprofen is a nonsteroidal anti-inflammatory drug and is often used as a model molecule to study such drug delivery systems. In the particular case of ibuprofen encapsulated in MCM-41, it is observed that the release profile in a gastric or intestinal simulated fluid is much faster that the bulk molecule. $^{14}$

From a pharmaceutical point of view, it is crucial to precisely characterize the physical state of a drug in the dosage form and solid state NMR became an essential tool for this 
purpose due to its ability to characterize (i) the physical state of the drug, (ii) the surface of the porous carrier and (iii) the interaction between the two. ${ }^{22}$ Recent literature showed that drugs confined in porous matrices are submitted to a confinement effect that influences their physical state at room temperature ${ }^{23,24,25,26,27}$. It is generally admitted that the exacerbated mobility evidenced in the case of encapsulated ibuprofen influences strongly the release profile observed in vitro. ${ }^{28,29}$ In particular, we showed in previous papers that such behavior must be taken into account for the NMR characterization of ibuprofen and model molecules (including benzoic acid) encapsulated in MCM-41. ${ }^{30,31,32,33}$ Indeed, due to the highly mobile behavior of the drug, the ${ }^{13} \mathrm{C}$ cross polarization (CP) NMR experiments on such solid samples are inefficient due to the partial averaging of heteronuclear ${ }^{1} \mathrm{H}-{ }^{13} \mathrm{C}$ dipolar interaction that prevents a high efficiency of the CP process, which is routinely used in solid state NMR.

In this contribution, we study ibuprofen and benzoic acid encapsulated in MCM-41 through the incipient wetness impregnation method by means of ${ }^{1} \mathrm{H}$ MAS NMR experiments in order to understand the molecules-silica interface. In particular, we use variable temperature experiments that allow highlighting the presence of water molecules trapped inside the pores. These molecules are part of a fast chemical exchange that takes place between the protons of the $\mathrm{COOH}$ group of the organic molecule and protons from water molecules at ambient temperature. This phenomenon influences the highly mobile behavior of the guest molecule and implies a weak interaction between the guest molecule and the silica surface that is related to the fast release profile of encapsulated ibuprofen observed in vitro. 


\section{Experimental}

MCM 41 synthesis, ibuprofen, benzoic acid and deuterated benzoic acid loading procedure.

The synthesis of Ibuprofen and benzoic acid encapsulated in MCM-41 was described in previous papers. ${ }^{30,32}$

Briefly, MCM-41 with a mean pore diameter of $30 \AA$ was obtained by mixing under stirring at room temperature $\mathrm{H}_{2} \mathrm{O}, \mathrm{NaOH}$, cetyltrimethylammonium bromide, and silica (Aerosil 200), in a given molar ratio of 20:0.25:0.1:1, respectively. Then the mixture was heated at $120{ }^{\circ} \mathrm{C}$ for $24 \mathrm{~h}$. The resulting white powders were filtered and plentifully washed with distilled water up to neutral $\mathrm{pH}$, then dried at $70{ }^{\circ} \mathrm{C}$ for $48 \mathrm{~h}$. The powders are then calcined at $600{ }^{\circ} \mathrm{C}$ for $6 \mathrm{~h}$ under air flux to remove the surfactant. Calcined MCM-41 powder was loaded with a solution of ibuprofen (Ibu), benzoic acid (BA) or deuterated benzoic acid (AB-D5) in ethanol $\left(\sim 0.310\right.$ mol. $\left.\mathrm{L}^{-1}\right)$ through the "incipient wetness impregnation" method previously described ${ }^{14}$. The deuterated benzoic acid is deuterated on the phenyl ring and of formula $\mathrm{C}_{6} \mathrm{D}_{5} \mathrm{COOH}$. Four successive impregnations of $0.500 \mathrm{~g}$ of $\mathrm{MCM}-41$ with a small amount of the solutions were performed. The solvent is removed between two impregnations by heating at $70{ }^{\circ} \mathrm{C}$ overnight. Samples are quickly washed with ethanol to remove the excess of crystallized organic molecules. The MCM-41 loaded ibuprofen, benzoic acid and deuterated benzoic acid samples will be referred in the forthcoming text as Ibu@MCM, BA@MCM and BA-D5@MCM.

The total amount of encapsulated organic molecules was determined through thermogravimetric analysis (TGA) on a SETARAM TG-DTA Instruments under air flow with a heating rate of $5{ }^{\circ} \mathrm{C} \cdot \mathrm{min}^{-1}$ up to $800{ }^{\circ} \mathrm{C}$ and was found to be $41.2,40.8$ and $48.9 \mathrm{w} \%$, for Ibu@MCM, BA@MCM and BA-D5@MCM, respectively (Figure S1). 
Humidity controlled samples were obtained by placing Ibu@MCM and BA@MCM one night in a close chamber with a saturated aqueous solution of $\mathrm{NaCl}$ corresponding to an atmosphere composed of $75 \%$ humidity.

\section{Solid state NMR}

Room temperature ${ }^{1} \mathrm{H}$ single pulse NMR experiments were performed on AV300 and AV400 Bruker spectrometers operating at $v\left({ }^{1} \mathrm{H}\right)=300$ and $400 \mathrm{MHz}$, respectively. Zirconia rotors of 4 or $2.5 \mathrm{~mm}$ diameters were spun at a MAS frequency ( $v_{\mathrm{MAS}}$ ) of 14 or $35 \mathrm{kHz}$, respectively. $\pi / 2$ pulses were 5 and $2.5 \mu \mathrm{s}$, respectively. Recycle delays (RD) were set to $2 \mathrm{~s}$. No temperature regulation was used for room temperature experiments.

Variable temperature experiments were performed on AV300 Bruker spectrometer using a $\mathrm{BCU}-\mathrm{Xtreme}$ Bruker accessory to regulate the temperature down to $-53{ }^{\circ} \mathrm{C}$. Zirconia rotors of $4 \mathrm{~mm}$ diameter were used and spun at $\mathrm{v}_{\mathrm{MAS}}=5 \mathrm{kHz} .{ }^{1} \mathrm{H}_{-}{ }^{29} \mathrm{Si}^{-1}{ }^{1} \mathrm{H}$ double "there and back" $\mathrm{CP}$ experiment consists of two consecutive cross polarization (CP) steps. The contact time of the first one from ${ }^{1} \mathrm{H}$ to ${ }^{29} \mathrm{Si}$ was set to $\mathrm{t}_{\mathrm{CP}} 1=10 \mathrm{~ms}$ and the second one from ${ }^{29} \mathrm{Si}$ to ${ }^{1} \mathrm{H}$ was set to $\mathrm{t}_{\mathrm{CP}} 2=1 \mathrm{~ms}$. Recycle delays $(\mathrm{RD})$ were set to $10 \mathrm{~s}$. Temperature calibration was achieved using lead nitrate $\mathrm{Pb}\left(\mathrm{NO}_{3}\right)_{2}$.

${ }^{13} \mathrm{C}$ cross polarization (CP) MAS were performed on a AV300 Bruker spectrometer using 4 $\mathrm{mm}$ rotors at $v_{\mathrm{MAS}}=5 \mathrm{kHz}$. Contact time was $5 \mathrm{~ms}$ and the recycle delay $3 \mathrm{~s}$.

${ }^{2} \mathrm{H}$ static experiments were recorded on a AV300 Bruker spectrometer using a quadrupolar echo sequence $(\pi / 2$ pulse $=3.6 \mu \mathrm{s}$ and delay $\tau=50 \mu \mathrm{s})$. The recycle delay was set to $60 \mathrm{~s}$ (solid) and 1s (liquid). 


\section{Results and discussion}

The ${ }^{1} \mathrm{H}$ MAS NMR spectra recorded at $9.4 \mathrm{~T}$ and $v_{\mathrm{MAS}}=35 \mathrm{kHz}$ of $\mathrm{Ibu}$ and Ibu@MCM are depicted in Figure 1. If the ${ }^{1} \mathrm{H}$ spectrum of pure ibuprofen is poorly resolved (that is common for an organic solid), in the case of Ibu@MCM, the ${ }^{1} \mathrm{H}$ resonances of each inequivalent proton are visible and individualized except for the $\mathrm{COOH}$ group which is absent as no resonances are observed at the expected chemical shift around $\delta\left({ }^{1} \mathrm{H}\right)=15 \mathrm{ppm}$ (see assignment in Figure 1). It is known that the ${ }^{1} \mathrm{H}$ MAS NMR spectrum of MCM-41 encapsulated ibuprofen is highly resolved at ambient temperature $\left(T_{\mathrm{amb}}\right)$. The spectrum is close to a liquid NMR spectrum although the sample is solid. It was demonstrated that this particular feature is due to the confinement effect that increases the dynamic of ibuprofen trapped inside the mesopores. This liquid-like behavior leads to the average of the ${ }^{1} \mathrm{H}-{ }^{1} \mathrm{H}$ homonuclear dipolar coupling that usually broaden the ${ }^{1} \mathrm{H}$ NMR signals in the solid state.

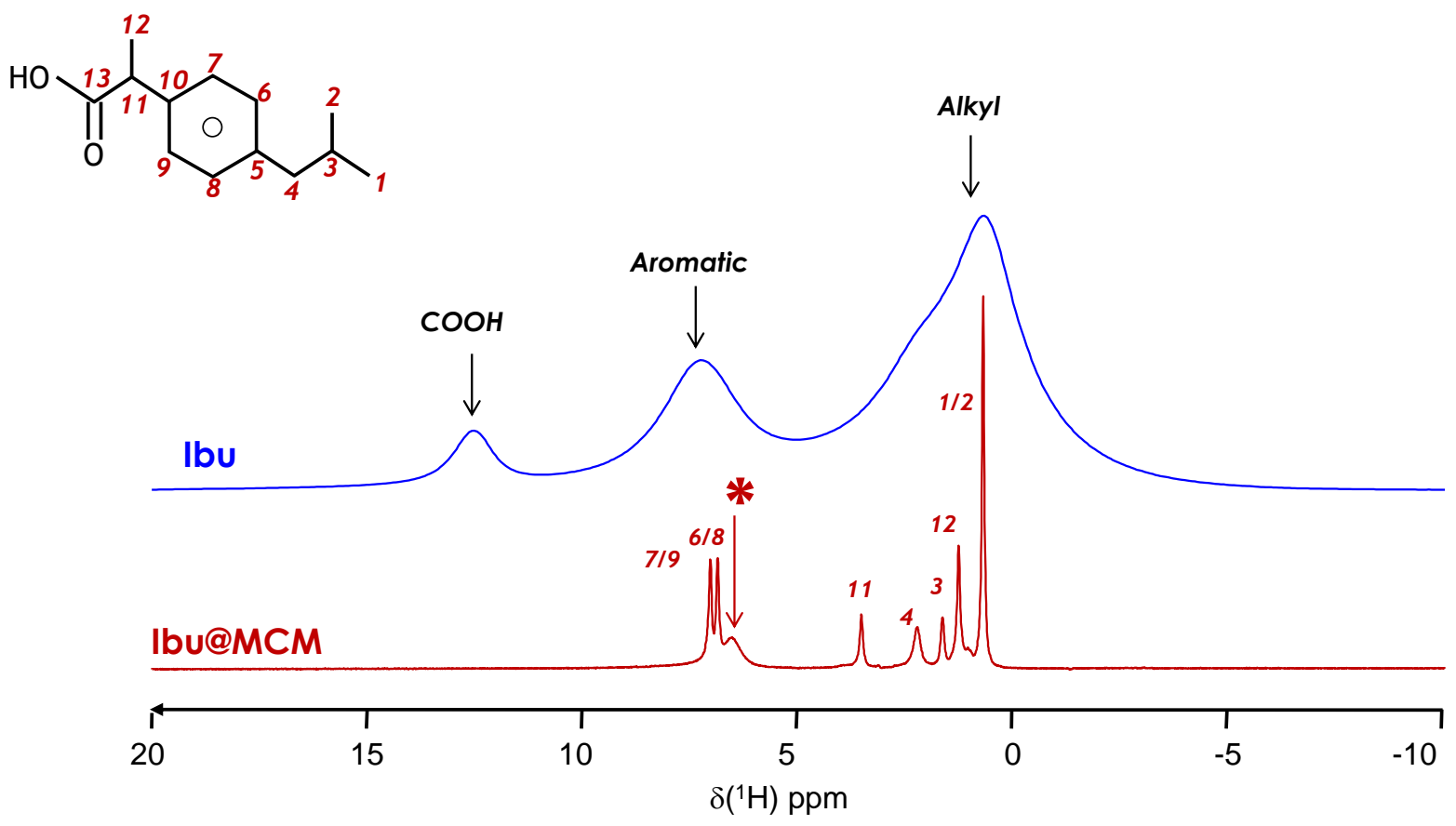


Figure 1. ${ }^{1}$ H MAS NMR spectra of Ibu and Ibu@ MCM together with the resonances assignment $\left(B_{0}=9.4 T, v_{M A S}=35 \mathrm{kHz}\right)$. Asterisk denotes the specific resonance studied through ${ }^{1}$ H MAS NMR variable temperature experiments (see text for details).

The absence of the proton resonance of the $\mathrm{COOH}$ group suggests either a deprotonated state of the molecule in the pores or a fast chemical exchange wherein the proton from the $\mathrm{COOH}$ group is involved. The second case was evidenced through already published variable temperature ${ }^{1} \mathrm{H}$ MAS NMR experiments. Below the glass transition $(\mathrm{Tg})$ of MCM-41 encapsulated ibuprofen $\left(\mathrm{Tg}=-50^{\circ} \mathrm{C}\right)$, the resonance of the $\mathrm{COOH}$ moiety is then observed at $\delta\left({ }^{1} \mathrm{H}\right)=15 \mathrm{ppm} .{ }^{30}$

Another specific resonance is observed in the ${ }^{1} \mathrm{H}$ MAS NMR spectrum of MCM-41 encapsulated ibuprofen at $T_{\mathrm{amb}}$ and not precisely assigned in the literature until now (asterisk in Figure 1). This resonance is broad and located at $6.7 \mathrm{ppm}$. It was shown through $2 \mathrm{D}{ }^{1} \mathrm{H}$ ${ }^{29} \mathrm{Si}$ HetCor experiments that this signal corresponds to protons close to silica sites. ${ }^{30}$ This specific signal is also observed for various MCM-41-encapsulated carboxylic acids samples and in particular in the case of benzoic acid ${ }^{31}$ that is often considered as a model molecule of ibuprofen due to the presence of common chemical moieties (phenyl ring and carboxylic acid group).

To investigate in details the absence of the $\mathrm{COOH}$ resonance due to fast chemical exchange phenomenon and a possible link with the specific broad resonance at $6.7 \mathrm{ppm}$, we performed variable temperature ${ }^{1} \mathrm{H}$ MAS NMR experiments. As it was shown that at low temperature the ${ }^{1} \mathrm{H}$ MAS NMR spectrum of Ibu@MCM is broad (due to the reduced mobility of the molecules and the reintroduction of the homonuclear ${ }^{1} \mathrm{H}-{ }^{1} \mathrm{H}$ dipolar coupling) and difficult to analyze in details, we used benzoic acid as a model molecule of ibuprofen, and in particular deuterated benzoic acid (BA-D5) encapsulated in MCM-41 (as unfortunately 
deuterated ibuprofen is not commercially available). For that latter sample only the phenyl ring is deuterated that allows removing the ${ }^{1} \mathrm{H}$ resonances from the phenyl ring and permit to follow easily the resonances of potentially exchangeable protons.

Figure 2 displays the ${ }^{1} \mathrm{H}$ MAS NMR spectra of BA and BA@MCM recorded at 7 T and $v_{\text {MAS }}=14 \mathrm{kHz}$. Similarly to the case of ibuprofen, if the ${ }^{1} \mathrm{H}$ NMR spectrum of BA is broad and poorly resolved, a highly resolved spectrum is obtained for BA@MCM where the resonances at 7.6, 7.0 and $6.9 \mathrm{ppm}$ are assigned to protons from the phenyl ring in ortho, para and meta position, respectively. ${ }^{32}$ The ${ }^{1} \mathrm{H}$ resonance from the $\mathrm{COOH}$ group is absent as for Ibu@MCM. A specific broad resonance at 8 ppm is also observed. Figure 2 displays also the ${ }^{1} \mathrm{H}$ MAS NMR spectrum of BA-D5@MCM recorded at $7 \mathrm{~T}, v_{\mathrm{MAS}}=5 \mathrm{kHz}$ and $\mathrm{T}=27^{\circ} \mathrm{C}$. As expected the spectrum displays only the latter resonance as the ${ }^{1} \mathrm{H}$ resonances from the phenyl ring are absent. 


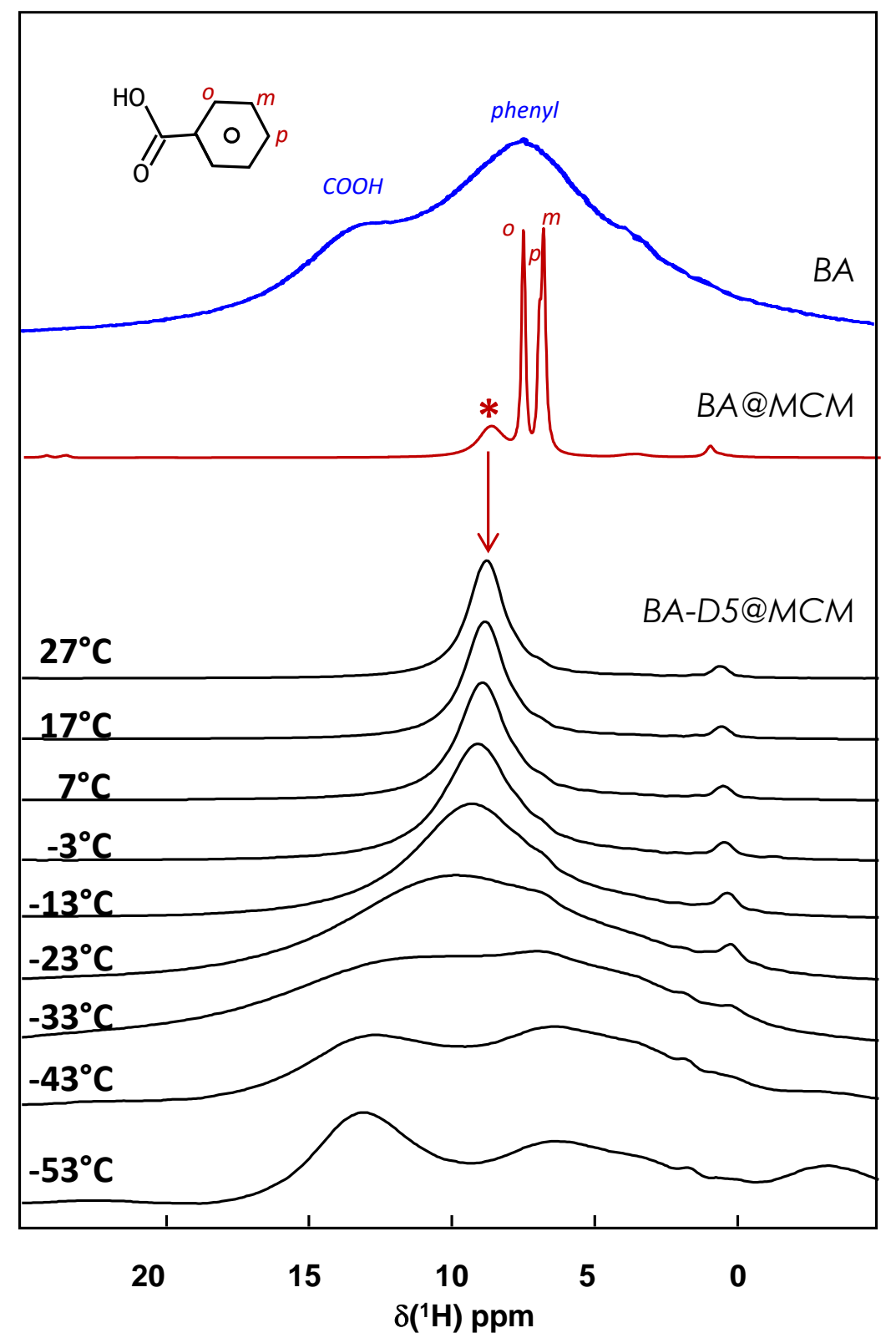

Figure 2. ${ }^{1} \mathrm{H}$ MAS NMR spectra of $B A\left(B_{0}=7 T, v_{M A S}=14 \mathrm{kHz}\right), B A @ M C M\left(B_{0}=7\right.$

$\left.T, v_{M A S}=14 \mathrm{kHz}\right)$ and BA-D5@MCM recorded at variable temperatures $\left(B_{0}=7 T, v_{M A S}=5\right.$

$\mathrm{kHz})$. Asterisk denotes the specific resonance followed through ${ }^{1} \mathrm{H}$ MAS NMR variable temperature experiments (see text for details). Resonances assignment for BA and BA@MCM is given in the Figure.

When decreasing the temperature from +27 to $-53^{\circ} \mathrm{C}$ (Figure 2), we observe the gradual broadening of that specific resonance which then split into two resonances at $-43^{\circ} \mathrm{C}$. At $-53^{\circ} \mathrm{C}$ the two signals are clearly distinct at $\delta\left({ }^{1} \mathrm{H}\right)=13$ and $6 \mathrm{ppm}$. We note that the glass 
transition temperature $(\mathrm{Tg})$ was determined through $\mathrm{DSC}$ measurement at $-55^{\circ} \mathrm{C}$ for BA@MCM of similar pore size. ${ }^{32}$ Thus, the protons corresponding to these two resonances are implied in a fast chemical exchange at $T_{\text {amb }}$ leading to the specific resonance at $8 \mathrm{ppm}$. At low temperature $(T \approx T \mathrm{~g})$, such chemical exchange is quenched. A relative proportion of $50 / 50$ for these two species is determined through the fitting of the spectrum. The coalescence of the two signals that happens between $-23^{\circ} \mathrm{C}$ and $-33^{\circ} \mathrm{C}$ allows the evaluation of the exchange rate constant $k$ and the activation free energy of the exchange process $\Delta G^{t}$. The exchange rate constant $k$ is calculated to $k=\pi \Delta v_{\mathrm{O}} / \sqrt{ } 2=4700 \mathrm{~Hz}$ where $\Delta v_{\mathrm{O}}$ is the frequency difference between the two exchanged sites. The activation free energy of the exchange process $\Delta G^{t}$ can be obtained from the following equation $\Delta G^{t}=R T_{C}\left[22.96+\ln \left(T_{C} / \Delta v_{0}\right)\right]$ (where $T_{C}$ is the coalescence temperature) and is found to be comprised between 43.30 and $41.49 \mathrm{~kJ} . \mathrm{mol}^{-1}$ (calculation for $T_{C}=-23$ and $-33^{\circ} \mathrm{C}$, respectively). This value is greater than the activation energy found for the intramolecular proton exchange in carboxylic acid dimers in the solid state where values around $4-5 \mathrm{~kJ} \mathrm{~mol}^{-1}$ have been determined. ${ }^{34}$ However it can be compared to proton exchange in $\mathrm{H}-\mathrm{Y}$ zeolite where the activation energy between hydroxyls from the supercage and from the sodalite cage is estimated to be $50 \mathrm{~kJ} . \mathrm{mol}^{-1} .^{35}$

The two resonances are assigned from the spectrum at $-53^{\circ} \mathrm{C}$ (Figure 3). The ${ }^{1} \mathrm{H}$ MAS NMR spectrum of BA-D5 reveals that the signal at $13 \mathrm{ppm}$ correspond to the $\mathrm{COOH}$ group as the chemical shifts is identical to the ${ }^{1} \mathrm{H}$ resonance in BA-D5. The $\delta\left({ }^{1} \mathrm{H}\right)$ being very sensitive to the strength of the H-bond in which the proton is engaged, ${ }^{36}$ it seems that at low temperature benzoic acid molecules are reorganized as dimers in the mesopores similarly to what exists in the crystalline state. ${ }^{37}$ 


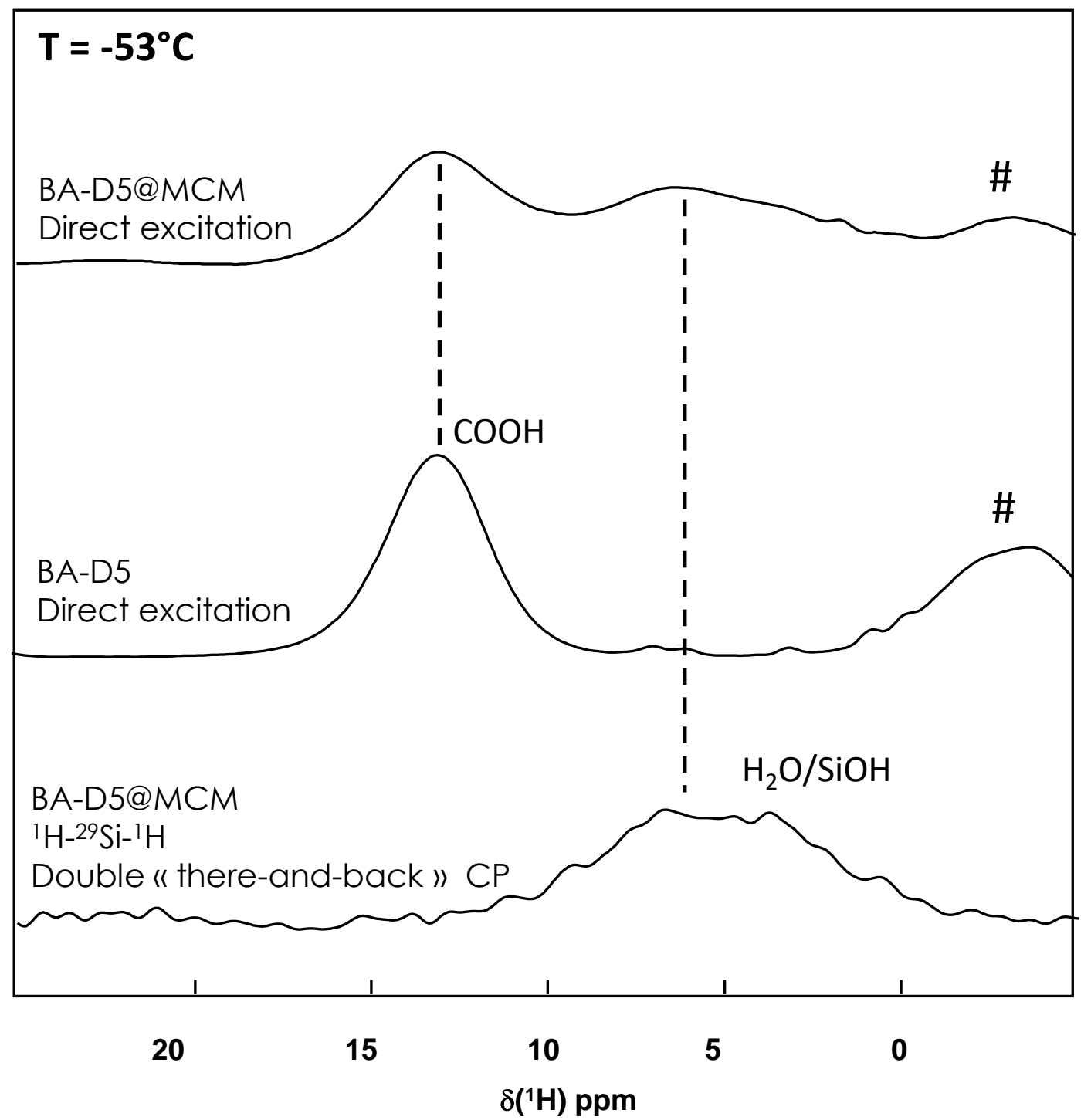

Figure 3. Low temperature $\left(T=-53^{\circ} \mathrm{C}\right){ }^{1} H$ MAS NMR spectra of BA-D5@MCM (direct excitation), BA-D5 (direct excitation) and BA-D5@MCM $\left({ }^{1} \mathrm{H}_{-}^{29}{ }^{2} \mathrm{Si}_{-}{ }^{1} \mathrm{H}\right.$ Double "there and back" CP). \# denotes spinning side bands. $B_{0}=7 \mathrm{~T}, v_{M A S}=5 \mathrm{kHz}$.

The second signal is assigned through a ${ }^{1} \mathrm{H}^{29}{ }^{29} \mathrm{Si}^{1}{ }^{1} \mathrm{H}$ Double "there-and-back" CP NMR experiment that consists in two consecutive $\mathrm{CP}$ transfers, from ${ }^{1} \mathrm{H}$ to ${ }^{29} \mathrm{Si}$ and then back to ${ }^{1} \mathrm{H} .{ }^{38}$ The first transfer is used to maximize the ${ }^{29} \mathrm{Si}$ magnetization (the corresponding contact time $\mathrm{t}_{\mathrm{CP}} 1$ is set to $10 \mathrm{~ms}$ ) and the second transfer, from ${ }^{29} \mathrm{Si}$ to ${ }^{1} \mathrm{H}$, allows the investigation of ${ }^{29} \mathrm{Si} \bullet \bullet \bullet{ }^{1} \mathrm{H}$ proximities by varying the corresponding contact time $\mathrm{t}_{\mathrm{CP}} 2$. For $\mathrm{t}_{\mathrm{CP}} 2=1 \mathrm{~ms}$, we observe the signal at $6 \mathrm{ppm}$ thus corresponding to protons bounded to the silica (Figure 3). 
According to the chemical shift, we assign this resonance to water molecules adsorbed on the silica surface through comparison with the work of Xu et al. ${ }^{39}$ that reports the ${ }^{1} \mathrm{H} \mathrm{NMR}$ spectra of activated MCM-41 at $183 \mathrm{~K}$ upon adsorption of water and deuterated water. Authors evidence a broad signal around $5.4 \mathrm{ppm}$ (similar to the ${ }^{1} \mathrm{H}$ signal that we evidence through ${ }^{1} \mathrm{H}^{-29}{ }^{29-}{ }^{1} \mathrm{H}$ Double “there-and-back” CP MAS experiment for BA-D5@MCM Figure 3) that they assign to "water hydrogen bonded to silanol groups". The amount of water molecules in our samples determined through TGA measurements (weight loss up to $120^{\circ} \mathrm{C}$ ) can be estimated to 4, 1.5 and 1.5 w\% for Ibu@MCM, BA@MCM and BA-D5@MCM, respectively (Figure S1). We note that these water molecules might correspond both to encapsulated water and water molecules at the surface of the silica particles. Nevertheless, the NMR result highlights that a significant part of these water molecules is trapped in the pores and takes part in the proton chemical exchange phenomenon with benzoic acid at ambient temperature. This ${ }^{1} \mathrm{H}$ signal assignment is confirmed by the ${ }^{1} \mathrm{H}$ spectra of BA and Ibu@ MCM equilibrated one night in a humidity controlled atmosphere $(75 \%$, thanks to a $\mathrm{NaCl}$ saturated aqueous solution) as the ${ }^{1} \mathrm{H}$ chemical shift of the specific resonance depends on that equilibration (Figure 4). At a high degree of humidity (75\%) this resonance is shifted down to $6 \mathrm{ppm}$, in the case of Ibu@MCM and down to around 5 ppm in the case of BA@MCM. As soon as the level of humidity is increased we observe a $\delta\left({ }^{1} \mathrm{H}\right)$ value closer to that of pure water, confirming the previous assignment and the crucial role of water molecules in the chemical exchange process. We observe that the position of the ${ }^{1} \mathrm{H}$ resonance for encapsulated samples depends on the recording conditions as the ${ }^{1} \mathrm{H}$ resonances are slightly shifted to higher chemical shifts when $v_{\text {MAS }}$ increases. Similarly, we believe that as soon as $v_{\text {MAS }}$ increases, the temperature of the sample increases and can lead to partial dehydration especially for long time NMR experiments if the rotor is not perfectly airtight. Moreover, we note that for BA@MCM actually two resonances at 5.1 and $4.7 \mathrm{ppm}$ are observed, that are 
related to water molecules inside and outside the pores of the mesoporous silica. ${ }^{27}$ These signals are much more intense than for Ibu@MCM that is an indication of the ease of water molecules to enter inside the mesopores which is higher for BA@ MCM than for Ibu@MCM. Finally, the water content inside the pores seems to influence the mobility of the molecules. In the case of ibuprofen, the ${ }^{1} \mathrm{H}$ line widths are sharper after equilibration in a water atmosphere which is the sign of an increased mobility.

According to the literature, ${ }^{27}$ diffusion of water or organic solvent vapors might drive the encapsulated molecules out of the porous network and lead to a recrystallization outside the pores. Nevertheless, ${ }^{1} \mathrm{H}$ spectra still display sharp resonances (Figure 4) evidencing that a high amount of molecules are submitted to confinement effect meaning that these molecules are still encapsulated into the pores. This finding is further confirmed by ${ }^{13} \mathrm{C} \mathrm{CP}$ MAS NMR experiments that highlight rigid species. The ${ }^{13} \mathrm{C} \mathrm{CP}$ MAS experiments of encapsulated molecules after one night equilibration in $75 \%$ humidity controlled atmosphere are not efficient (poor $\mathrm{S} / \mathrm{N}$ ) due to the rapid reorientation of the molecules (Figure S2). Nevertheless, some typical resonances of crystalline ibuprofen $\left(\mathrm{COOH}\right.$ and $\mathrm{CH}_{3}$ from the isopropyl group see arrows) are evidenced. From the ${ }^{1} \mathrm{H}$ MAS and ${ }^{13} \mathrm{C}$ CP MAS experiments we can conclude that, if some molecules have recrystallized outside of the pores, this amount is low compared to the encapsulated one. 


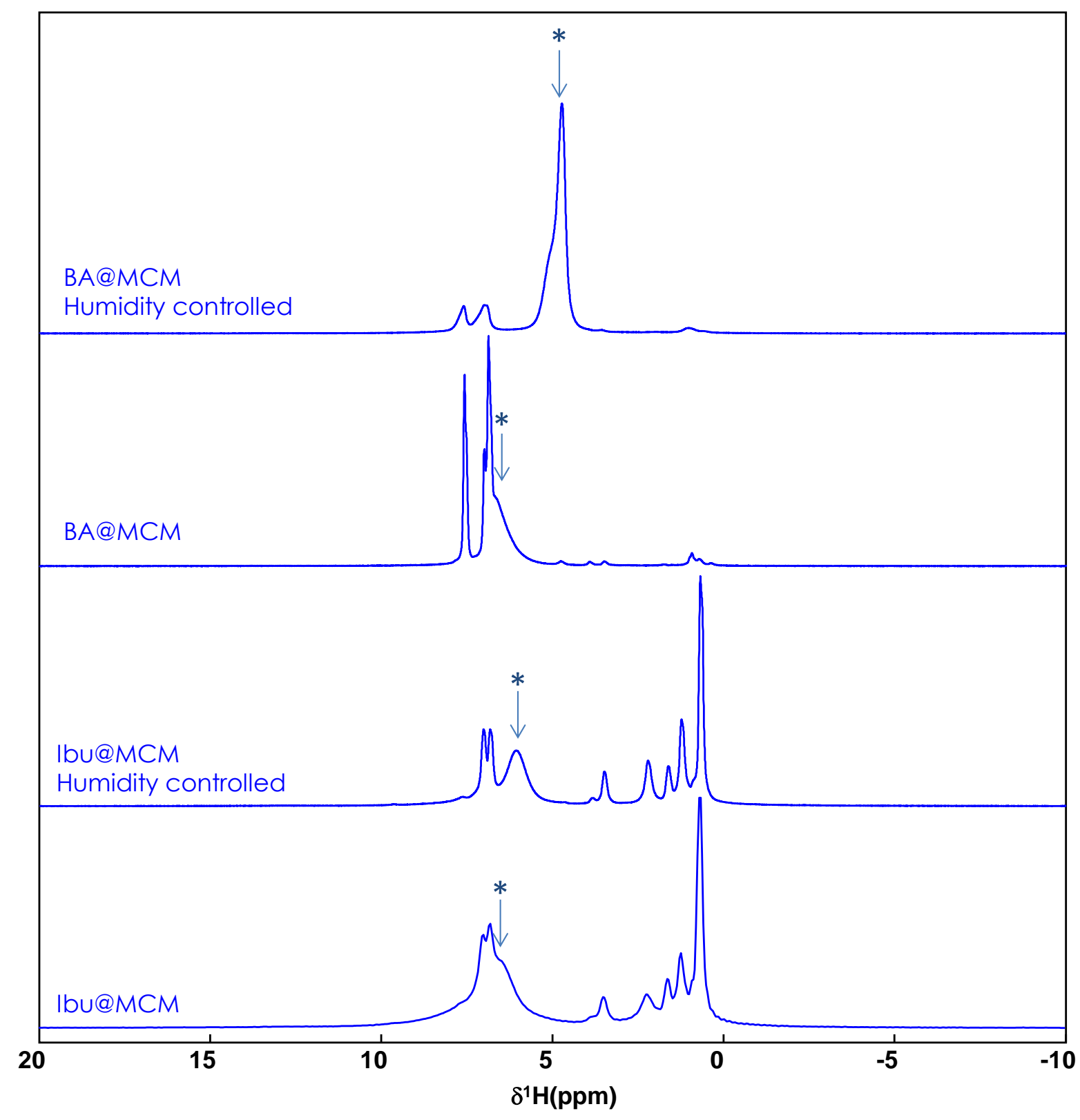

Figure 4. ${ }^{1} H$ MAS NMR spectra of BA@MCM and Ibu@MCM without and with one night of equilibration in a 75\% humidity controlled atmosphere. Asterisks denote the $\mathrm{H}_{2} \mathrm{O}$ COOH exchange resonance. $B_{0}=7 \mathrm{~T}, v_{M A S}=14 \mathrm{kHz}$.

${ }^{2} \mathrm{H}$ NMR spectroscopy is a pertinent tool to study dynamics of solids and encapsulated molecules in particular. ${ }^{40} \mathrm{We}$ recorded variable temperature ${ }^{2} \mathrm{H}$ static NMR experiments to explore the dynamical behavior of encapsulated benzoic acid (BA-D5@MCM) and compared 
to the pure substance (BA-D5) (Figure S3). At ambient temperature the ${ }^{2} \mathrm{H}$ static NMR spectrum of BA-D5@MCM exhibits a single resonance revealing a rapid isotropic reorientation of the molecules confirming the "liquid-like" behavior of benzoic acid when encapsulated in silica mesopores. ${ }^{32}$ The linewidth of the resonance increases as the temperature decreases down to $-15^{\circ} \mathrm{C}$ probably due to a decrease of the mobility implying a decrease of the tranverse relaxation time $\mathrm{T}_{2}$. This rapid reorientation is quenched between -15 and $-30^{\circ} \mathrm{C}$ in agreement with the variable temperature ${ }^{1} \mathrm{H}$ NMR experiments depicted in Figure 2 leading to a "solid-like" ${ }^{2} \mathrm{H}$ NMR spectrum. The quadrupolar parameters can be extracted through the fitting of the ${ }^{2} \mathrm{H}$ quadrupolar lineshape $\left(v_{\mathrm{Q}}=263 \mathrm{kHz}\right.$ et $\eta_{\mathrm{Q}}=0.02$ at $\mathrm{T}$ $\left.=-50^{\circ} \mathrm{C}\right)$ and are similar to bulk BA-D5 $\left(v_{\mathrm{Q}}=269 \mathrm{kHz}\right.$ et $\eta_{\mathrm{Q}}=0.04$ à $\left.\mathrm{T}=-53^{\circ} \mathrm{C}\right)$. In the case of bulk BA-D5, we note that the melting point is estimated to $117^{\circ} \mathrm{C}$ in our experimental conditions, in good agreement with the tabulated value of $122.4^{\circ} \mathrm{C} .{ }^{41}$

We showed in a previous work that the dynamical behavior of encapsulated ibuprofen do not strictly followed the Gibbs-Thomson model for which the $T \mathrm{~g}$ is inversely proportional to the pore size as similar values are found both for ibuprofen $\left(T \mathrm{~g}=-55^{\circ} \mathrm{C}\right)$ and benzoic acid $\left(T \mathrm{~g}=-58^{\circ} \mathrm{C}\right)^{32}$ entrapped in mesoporous silica with two different pore size of 30 and $100 \AA$. This implies that in addition to physical confinement, there is a chemical interaction between the molecule and the silica surface. ${ }^{1}$ Our experimental work highlights this specific interaction that involves water molecules at the silica surface and is in agreement with recent work based on DFT calculations showing that $(i)$ water molecules have a high affinity with hydroxylated amorphous silica surface and (ii) that when Ibu and BA molecules are adsorbed onto a microsolvated silica surface they exhibit a mobile behavior through dynamical H-bonds between the water layer and the organic molecule. ${ }^{42}$

Figure 5 summarizes the dynamical behavior of ibuprofen encapsulated in MCM-41 at $T_{\text {amb }}$ that is actually related to two phenomena: $(i)$ the fast chemical exchange between the 
protons of $\mathrm{COOH}$ groups and the water molecules (red arrows) and (ii) the rapid reorientation of the molecule due to the confinement effect (green arrows). In addition to these dynamical phenomena, a translational mobility of ibuprofen along the mesopores of MCM-41 was recently evidenced through PFG-MAS NMR studies. ${ }^{43}$

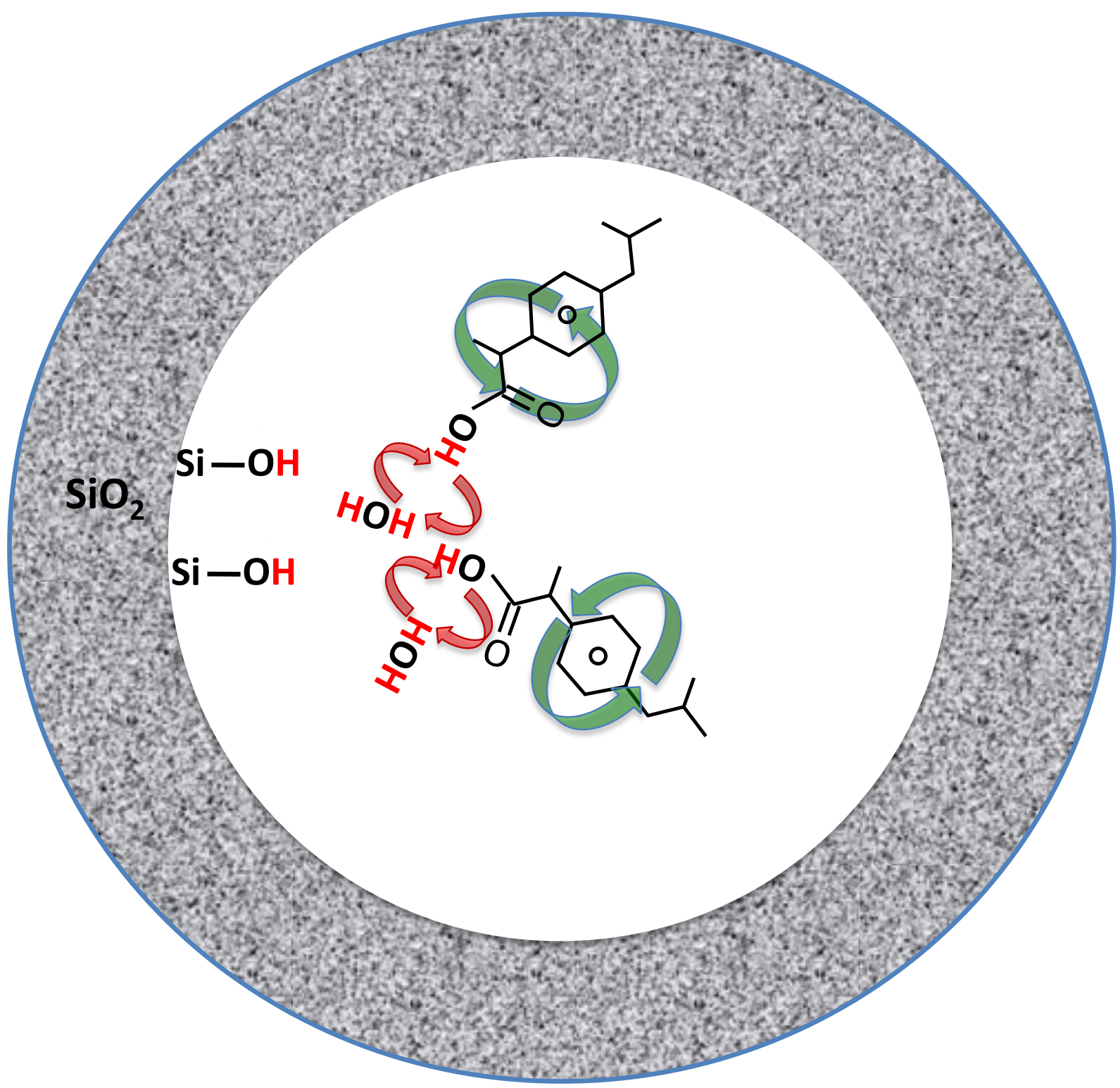

Figure 5. Schematic representation of the dynamical behavior of ibuprofen (or

benzoic acid) at ambient temperature inside the mesopores of MCM-41. Red circular arrows represent the fast chemical exchange between the protons of $\mathrm{COOH}$ groups and the water molecules. Green circular arrows represent the rapid reorientation of the molecule due to the 
confinement effect (the relative proportion of water $v$ ibuprofen or benzoic acid molecules is not known).

\section{Conclusion}

In this contribution, we demonstrated through variable temperature ${ }^{1} \mathrm{H}$ MAS NMR experiments that, in addition to confinement effect, the specific dynamical behavior of ibuprofen and benzoic acid encapsulated in MCM-41 is related to a proton fast chemical exchange that takes place at ambient temperature between the $\mathrm{COOH}$ group of the organic molecule and water molecules. Below $-40^{\circ} \mathrm{C}$ this fast exchange is quenched for benzoic acid and the molecules rearrange under the form of dimer.

In conclusion, we believe that these results are of primary importance because:

(i) They help to understand the rapid release kinetics of ibuprofen observed in vitro. Indeed, the host-guest interaction and the guest-guest interaction are of weak intensity due to both the physical confinement and the chemical exchange with water molecules present at the silica surface. This probably leads to a more rapid solubilization compared to pure and crystalline ibuprofen and increases its bioavailability.

(ii) More generally, they show the importance of water molecules at the silica surface while they are often overlooked. Indeed, in the case of small organic encapsulated molecules, the silica/organic interface is often featured through a direct and rigid interaction between a $\mathrm{SiOH}$ group and a functional group of the organic molecule. 
Concerning the presence of water molecules, it is difficult to state on their origin. Indeed, mesoporous silicas are "activated" (heating at $200^{\circ} \mathrm{C}$ in vacuum to desorb water molecules) before the encapsulation process. Nevertheless, several hypotheses may be advanced: it is possible that $(i)$ the procedure is not sufficient to remove all the water molecules; (ii) silica readsorb water molecules from the atmosphere very quickly before encapsulation and finally (iii) the residual water molecules are present in the ethanol solution used for the encapsulation. Anyway, very interestingly, they seem to be critical in the release properties. These hypotheses are specific to the incipient wetness impregnation method employed in this study. Using the melting method for the encapsulation step would lead to a reduced amount of residual water molecules.

\section{Author information}

Corresponding author

*E-mail: thierry.azais@upmc.fr

Present addresses

$\dagger$ a Centro de Reconocimiento Molecular y Desarrollo Tecnológico (IDM), Unidad Mixta

Universitat Politècnica de València, Universitat de València, Spain.

b Departamento de Química, Universitat Politècnica de València, Camino de Vera s/n E46022 Valencia, Spain.

\section{Acknowledgment}

This work was financially supported by the Emergence-UPMC-2009 research program from UPMC Univ Paris 06. 


\section{Supporting Information}

TGA measurements, ${ }^{13} \mathrm{C} \mathrm{CP}$ MAS and variable temperature ${ }^{2} \mathrm{H}$ static NMR spectra are provided as Supporting Information. 


\title{
References
}

\begin{abstract}
${ }^{1}$ Alcoutalbi, M.; McKenna, G. B. Effects of confinement on material behaviour at the nanometre size scale. $J$. Phys.: Condens. Matter 2005,17, R461-R524.

${ }^{2}$ Alba-Simionesco, C.; Coasne, B.; Dosseh, G.; Dudziak, G.; Gubbins, K E.; Radhakrishnan, R.; SliwinskaBartkowiak, M. Effects of confinement on freezing and melting. J. Phys.: Condens. Matter 2006, 18, R15-R68. ${ }^{3}$ Patrick, W. A.; Kemper, W. A. Melting temperatures of compounds adsorbed on silica gel1. J. Phys. Chem. 1938, 42, 369.
\end{abstract}

${ }^{4}$ Rennie, G. K.; Clifford, J. Melting of ice in porous solids. J. Chem. Soc. 1977, 73, 680.

${ }^{5}$ Morishige, K.; Kawano, K. Freezing and melting of methanol in a single cylindrical pore: dynamical supercooling and vitrification of methanol. J. Chem. Phys. 2000, 112, 11023-11029.

${ }^{6}$ Morineau, D.; Guégan, R.; Xia, Y.; Alba-Simionesco, C. Structure of liquid and glassy methanol confined in cylindrical pores. J. Chem. Phys. 2004, 121, 1466-1473.

${ }^{7}$ Takei, T.; Konishi, T.; Fuji, M.; Watanabe, T.; Chikazawa, M. Phase transition of capillary condensed liquids in porous silica: effect of surface hydroxyl groups. Thermochim. Acta 1995, 267, 159-167.

${ }^{8}$ Watanabe, A.; Iiyama, T.; Kaneko, T. Melting temperature elevation of benzene confined in graphitic micropores. Chem. Phys. Lett. 1999, 305, 71-74.

${ }^{9}$ Gedat, E.; Schreiber, A.; Albrecht, J.; Emmler, T.; Shenderovich, I.; Findenegg, G. H.; Limbach, H.-H.; Buntkowsky, G. ${ }^{2}$ H-solid-state NMR study of benzene-d6 confined in mesoporous silica SBA-15. J. Phys. Chem. B 2002, 106, 1977-1984.

${ }^{10}$ Dosseh, G.; Xia, Y.; Alba-Simionesco, C. Cyclohexane and benzene confined in MCM-41 and SBA-15: confinement effects on freezing and melting. J. Phys. Chem. B 2003, 107, 6445-6453.

${ }^{11}$ Aksnes, D. W.; Kimtys, L. ${ }^{1} \mathrm{H}$ and ${ }^{2} \mathrm{H}$ NMR studies of benzene confined in porous solids: melting point depression and pore size distribution. Solid State Nucl. Magn. Reson. 2004, 25, 146-152.

12 Vallet-Regi, M.; Ramila, A.; del Real, R. P.; Pérez-Pariente, J. A new property of MCM-41: drug delivery system. Chem. Mater. 2001, 13, 308-311.

${ }^{13}$ Vallet-Regi, M.; Balas, F.; Arcos, D. Mesoporous materials for drug delivery. Angew. Chem., Int. Ed. 2007, 46, 7548-7558.

${ }^{14}$ Charnay, C.; Begu, S.; Tourne-Peteilh, C.; Nicole, L.; Lerner, D. A.; Devoisselle, J.-M. Inclusion of ibuprofen in mesoporous templated silica: drug loading and release property. Eur. J. Pharm. Biopharm. 2004, 57, 533-540.

${ }^{15}$ Andersson, J.; Rosenholm, J.; Areva, S.; Lindén, M. Influences of material characteristics on ibuprofen drug loading and release profiles from ordered micro-and mesoporous silica matrices. Chem. Mater. 2004, 16, 41604167.

${ }^{16}$ Horcajada, P.; Ramila, A.; Pérez-Pariente, J.; Vallet-Regi, M. Influence of pore size of MCM-41 matrices on drug delivery rate. Microporous Mesoporous Mater. 2004, 68, 105-109.

${ }^{17}$ Andersson, J.; Areva, S.; Spliethoff, B.; Lindén, M. Sol-gel synthesis of a multifunctional, hierarchically porous silica/apatite composite. Biomaterials 2005, 26, 6827-6835.

${ }^{18}$ Qu, F.; Zhu, G.; Lin, H.; Sun, J.; Zhang, D.; Li, S.; Qiu, S. Drug self-templated synthesis of ibuprofen/mesoporous silica for sustained release. Eur. J. Inorg. Chem. 2006, 3943-3947.

${ }^{19}$ Heikkilä, T.; Salonen, J.; Tuura, J.; Hamdy, M. S.; Mul, G.; Kumar, N.; Salmi, T.; Yu Murzin, D.; Laitinen, L.; Kaukonen, A. M. et al. Mesoporous silica material TUD-1 as a drug delivery system. Int. J. Pharm. 2007, $331,133-138$.

${ }^{20}$ Aiello, R.; Cavallaro, G.; Giammona, G.; Pasqua, L.; Pierro, P.; Testa, F. Mesoporous silicate as matrix for drug delivery systems of non-steroidal antinflammatory drugs. Stud. Surf. Sci. Catal. 2002, 142, 1165-1172.

${ }^{21}$ Munoz, B.; Ramila, A.; Perez-Pariente, J.; Diaz, I.; Vallet-Regi, M. MCM-41 organic modification as drug delivery rate regulator. Chem. Mater. 2003, 15, 500-503.

${ }^{22}$ Skorupska, E.; Jeziorna, A.; Kazmierski, S.; Potrzebowski, M. J. Recent progress in solid-state NMR studies ofdrugs confined within drug delivery systems. Solid State Nucl. Magn. Reson. 2014, 57-58, 2-16

${ }^{23}$ Babonneau, F.; Yeung, L.; Steunou, N.; Gervais, C.; Ramila, A.; Vallet-Regi, M. Solid state NMR characterisation of encapsulated molecules in mesoporous silica. J. Sol-Gel Sci. Technol. 2004, 31, $219-223$. ${ }^{24}$ Lehto, V. P.; Vähä-Heikkilä, K.; Paski, J.; Salonen, J. Use of thermoanalytical methods in quantification of drug load in mesoporous silicon microparticles. J. Therm. Anal. Calorim. 2005, 80, 393.

${ }^{25}$ Tang, X.-P.; Cheong Ng, N.; Nguyen, H.; Mogilevski, G.; Wu, Y. The molecular dynamics and melting transition of the confined ibuprofen in titania nanotube studied by NMR. Chem. Phys. Lett. 2008, 452, $289-295$.

${ }^{26}$ Riikonen, J.; Mäkilä, E.; Salonen, J.; Lehto, V.-P. Determination of the physical state of drug molecules in mesoporous silicon with different surface chemistries. Langmuir 2009, 25, 6137-6142.

${ }^{27}$ Skorupska, E.; Jeziorna, A.; Paluch,P.; Potrzebowski, M. J. Ibuprofen in mesopores of mobil crystalline material 41 (MCM-41): a deeper understanding. Mol. Pharmaceutics 2014, 11, 1512-1519. 
${ }^{28}$ Aerts, C. A.; Verraedt, E.; Mellaerts, R.; Depla, A.; Augustijns, P.; Van Humbeeck, J.; Van den Mooter, G.; Martens, J. A. Tunability of pore diameter and particle size of amorphous microporous silica for diffusive controlled release of drug compounds. J. Phys. Chem. C 2007, 111, 13404-13409.

${ }^{29}$ Mellaerts, R.; Jammaer, J. A. G.; Van Speybroeck, M.; Chen, H.; Van Humbeeck, J.; Augustijns, P.; Van den Mooter, G.; Martens, J. A. Physical state of poorly water soluble therapeutic molecules loaded into SBA-15 ordered mesoporous silica carriers: a case study with itraconazole and ibuprofen. Langmuir 2008, 24, 86518659 .

${ }^{30}$ Azaïs, T.; Tourné-Pétheil, C.; Aussenac, F.; Baccile, N.; Coelho, C.; Devoisselle, J.-M.; Babonneau, F. Solidstate NMR study of ibuprofen confined in MCM-41 material. Chem. Mater. 2006, 18, 6382-6390.

${ }^{31}$ Azaiis, T.; Hartmeyer, G.; Quignard, S.; Laurent, G.; Tourné-Péteilh, C.; Devoisselle, J-M.; Babonneau, F. Solid-state NMR characterization of drug-model molecules encapsulated in MCM-41 silica. Pure Appl. Chem. 2009, 81 1345-1355.

${ }^{32}$ Azaïs, T.; Hartmeyer, G.; Quignard, S.; Laurent, G.; Babonneau, F. Solution state NMR techniques applied to solid state samples: characterization of benzoic acid confined in MCM-41. J. Phys. Chem. C 2010, 114, 8884 8891.

${ }^{33}$ Aiello, D.; Folliet, N.; Laurent, G.; Testa, F.; Gervais, C.; Babonneau, F.; Azaïs, T. Solid state NMR characterization of phenylphosphonic acid encapsulated in SBA-15 and aminopropyl-modified SBA-15. Microporous Mesoporous Mater. 2013, 166, 109-116.

${ }^{34}$ Meier, B. H.; Graf, F. and Ernst, R.R. Structure and dynamics of intramolecular hydrogen bonds in carboxylic acid dimers: a solid state NMR study. J. Chem. Phys. 1982, 76, 767-773.

${ }^{35}$ Asakawa, N; Motokura, K.; Yashima, T.; Koyama, T.; Onuki, T.; Miyaji, A. and Baba, T. Proton exchange reaction between hydroxyl groups in the supercage and those in the sodalitecage of $\mathrm{Y}$ zeolite as studied by variable temperature ${ }^{1}$ H MAS NMR. J. Phys. Chem. C. 2012, 116, 17734-17738.

${ }^{36}$ Gervais, C.; Coelho, C.; Azais, T.; Maquet, J.; Laurent, G.; Pourpoint, F.; Bonhomme, C.; Florian, P.; Alonso, B.; Guererro, G. et al. First principles NMR calculations of phenylphosphinic acid $\mathrm{C}_{6} \mathrm{H}_{5} \mathrm{HPO}(\mathrm{OH})$ : Assignments, orientation of tensors by local field experiments and effect of molecular motion. J. Magn. Reson. 2007, 187, 131-140.

${ }^{37}$ Freer, A. A.; Bunyan, J. M.; Shankland, N.; Sheen, D. B. Structure of (S)-(+)-ibuprofen. Acta Crystallogr., Sect. C 1993, 49, 1378.

${ }^{38}$ Folliet, N.; Roiland, C.; Bégu, S.; Aubert, A.; Mineva, T.; Goursot, A.; Selvaraj, K.; Duma, L.; Tielens, F.; Mauri, F. et al. Investigation of the interface in silica-encapsulated liposomes by combining solid state NMR and first principles calculations. J. Am. Chem. Soc. 2011, 133, 16815-16827.

${ }^{39}$ Xu, M.; Harris, K. D. M. and Meurig Thomas, J. Mapping the evolution of adsorption of water in nanoporous silica by in situ solid-state ${ }^{1} \mathrm{H}$ NMR spectroscopy. J. Am. Chem. Soc. 2008, 130, 5880-5882.

${ }^{40}$ Albunia, A. R.; Graf, R.; Grassi, A.; Guerra, G. and Spiess, H. W. Geometry of Complex Molecular Motions of Guest Molecules in Polymers from Solid State ${ }^{2}$ H NMR Macromolecules 2009, 42, 4929-4931.

${ }^{41}$ Haynes, W.M. Handbook of Chemistry and Physics. CRC Press Inc.: Boca Raton, FL, 2010-2011.

${ }^{42}$ Tielens, F.; Folliet, N.; Bondaz, L.; Etemovic, S. Babonneau, F.; Gervais, C. and Azaïs, T. Molecular picture of the adsorption of ibuprofen and benzoic acid on hydrated amorphous silica through DFT-D calculations combined with solid-state NMR experiments. J. Phys. Chem. C 2017, 121, 17339-17347.

${ }^{43}$ Guenneau, F.; Kuldeep Panesar, K.; Nossov, A.; Marie-Anne Springuel-Huet, M-A.; Azaïs, T.; Babonneau, F.; Tourné-Péteilh, C.; Devoisselle, J-M.; Gédéon, A. Probing the mobility of ibuprofen confined in MCM-41 materials using MAS-PFG NMR and hyperpolarised- ${ }^{129}$ Xe NMR spectroscopy. Phys. Chem. Chem. Phys. 2013, $15,18805-18808$. 
TOC Graphic

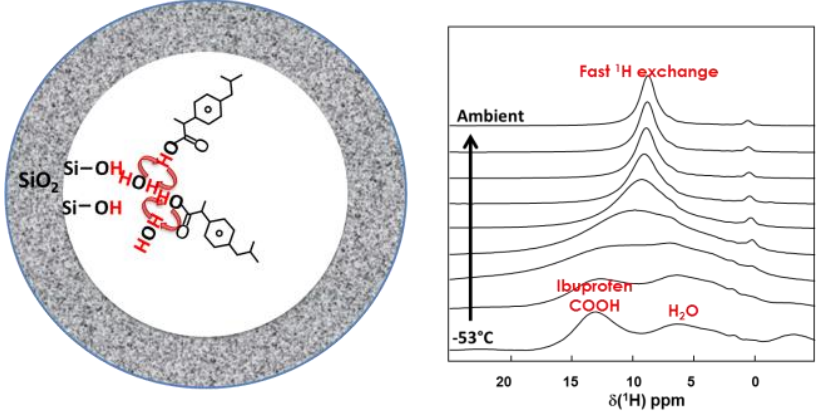

